\title{
Johann Jakob Wepfers Doktordisputation über das Herzklopfen (1647)
}

\author{
Von Nikolaus Mani
}

Wepfers Inauguraldisputation «De palpitatione cordis» wurde 1647 in Basel gedruckt $^{1}$. Die Abhandlung befaßt sich zunächst mit einem durchaus konventionellen und im universitären Bereich seit Jahrhunderten immer wieder disputierten Thema. Die Bearbeitung und Durchforstung dieses alten Problems zeigt indessen unverkennbar die Geisteshaltung und Methode des späteren Schaffens von Wepfer. Es ist das klare Bekenntnis zur neuen anatomisch-physiologischen Forschungs- und Denkweise, die Einsicht in die fundamentale Tragweite des Blutkreislaufs, der Physiologie und Pathologie revolutioniert, und wir erkennen schon in dieser frühen Schrift Wepfers erstaunliches Vermögen, klinische Probleme auf dem Boden der anatomisch-physiologischen Grundlagenforschung in völlig neuer Weise zu erkennen und zu klären.

Die gründlichen, aus der Schule von Heinrich Buess hervorgehenden Untersuchungen Pietro Eichenbergers ${ }^{2}$ über Leben und Werk Wepfers zeigen eindrücklich die große Wirkung von Wepfers dreijährigem Studienaufenthalt in Padua für die Ausreifung seiner medizinischen Denkweise. In Padua wurde er auf das stärkste von Johann Vesling ${ }^{3}$ und dessen Studentenkreis beeinflußt. Hier wurde Wepfer endgültig von der unumstößlichen Wahrheit des motus circularis Harveys überzeugt; hier traf er auch Thomas Bartholin ${ }^{4}$, der als einflußreicher früher Vertreter und Propagator des Blutkreislaufs und der experimentellen Forschungsmethode Wepfer nachhaltig beeinflußte.

Nach der Rückkehr aus Padua veröffentlichte Wepfer seine Doktorrede über das Herzklopfen, die eine reiche Frucht seines Aufenthaltes an der berühmten anatomischen Forschungsstätte Norditaliens bildet. Zunächst berichtet Wepfer über antike und zeitgenössische Erklärungen des Herzklopfens. Galen hatte in seinem Buch «De tremore, palpitatione, convulsione et rigore» als Ursache des Herzklopfens einen dichten und dampfartigen Spiritus verantwortlich gemacht, der aus dem Herzen nicht entweichen könne und zur Palpitatio cordis führe ${ }^{5}$. Daniel Sennert hatte in seinen «Institutiones Medicinae» angegeben, daß das Herz bei Perzeption von lästigen Stoffen zu mächtigen Bewegungen angetrieben wird ${ }^{6}$. Diese Vor- 
stellung geht, wie Owsei Temkin in seiner klassischen Studie über Glissons Lehre der Irritation dargelegt hat, auf galenische Quellen zurück ${ }^{7}$. Nach Galen werden kontraktile Organe wie Magen, Darm oder Blase durch beißenden Inhalt, durch Überfüllung oder durch Ausdehnung zur Kontraktion angeregt ${ }^{8}$. Aufgrund dieser galenischen Reiztheorie finden wir in der ersten Hälfte des 17. Jahrhunderts folgende geläufige Vorstellung über das Herzklopfen. Ursache der Palpitatio cordis ist eine Stimulierung der kardialen Pulsationskraft durch Entzündungen, Tumoren, böse Säfte oder verdorbene Spiritus. Wepfer erweiterte und konkretisierte diese Ansicht auf dem Boden des motus circularis sanguinis und mit Befunden aus der experimentellen Physiologie und pathologischen Anatomie. Herzklopfen, so stellt Wepfer fest, rührt nicht von einer gewaltsamen Ausdehnung des Herzens durch dicke Dämpfe her ${ }^{9}$. Es besteht vielmehr in einer krankhaften, heftigen, schnellen und häufigen Bewegung des Herzens; diese entsteht bei Reizung der kontraktilen Kraft des Herzens durch eine lästige Materie. Dabei sucht das Herz diese Materie auszustoßen ${ }^{10}$. Beim Herzklopfen erfolgt eine kräftige Verkürzung der Längs- und Querfasern des Herzens. Die Herzhöhlen werden dadurch verengt, wobei die Herzspitze unterhalb der linken Brustwarze an die Brustwand stößt. Wepfer beruft sich hier auf die Untersuchungsergebnisse der berühmten Anatomen Realdo Colombo und William Harvey. Wepfer führt auch experimentelle Befunde an. Schneidet man beim lebenden Tier die Herzspitze weg, so kann man die systolische Austreibung des Blutes gut beobachten. Legt man bei einem Menschen, der sich über Herzklopfen beklagt, die Hand unter die linke Brustwarze, so spürt man einen kräftigen Herzstoß. Wenn man bei Fröschen das Herz bloßlegt, sieht man, wie die Herzspitze bei der Systole auf die Basis zugeht und sich zugleich der Brustwand nähert ${ }^{11}$. Das spezifische Substrat der Palpitation, so hält Wepfer fest, liegt in den kontraktilen Herzfasern. Auch ohne Verbindung mit dem Nervensystem, z. B. am ausgeschnittenen Herzen ${ }^{12}$, ziehen sich dessen Fasern zusammen. Dies läßt sich am Herzen von Fischen und Vierfüßlern beobachten. Bedingungen für das Weiterschlagen des exzidierten und isolierten Herzens sind $W$ ärme und Unversehrtheit der Herzfasern ${ }^{13}$. Das Herz besitzt das Vermögen (potentia), schädigende Einflüsse zu empfinden (agnoscere); dies geschieht durch den Tastsinn und wird vielleicht durch die Herznerven ermöglicht. Auf jeden Fall weisen vivisektorische Versuche auf ein Empfindungsvermögen des Herzens hin. Wird das Herz am lebenden Tier bloßgelegt und mit einer Nadel angestochen, antwortet es mit kräftigen Kontraktionen ${ }^{14}$. Für Wepfer besteht das 
Herzklopfen aus intensiven Herzzuckungen, die durch fortdauernde auf das Herz einwirkende schädliche Reize (irritamentum, incitamentum, stimulus adversus, res molesta) bewirkt werden ${ }^{15}$. Das Herz versucht dabei, diese schädlichen Einwirkungen durch kräftige Pulsschläge zu entfernen. Diese schädlichen Bedingungen bilden die unmittelbare Ursache (causa proxima) des Herzklopfens und können verschiedener Art sein: eine übermäßige, das Herz belastende Blutmenge, sodann Hindernisse, die dem Ausströmen des Blutes im Wege stehen, z. B. Blutkoagula oder fleischige Auswüchse an der Herzbasis, schließlich auch scharfe Körpersäfte ${ }^{16}$.

Die Inauguraldissertation Wepfers über das Herzklopfen zeigt folgende Elemente, die auch für die späteren Leistungen Wepfers charakteristisch sind. Wepfer ist bestrebt, die klinischen Erscheinungen auf dem Boden der Anatomie, der Pathomorphologie und der experimentellen Physiologie zu erklären. Er sucht vor allem die unmittelbaren Krankheitsursachen zu erkennen, in diesem Falle die pathologischen Bedingungen, die zur Irritation der Herzfasern führen. Im Zentrum der Lehre Wepfers über das Herzklopfen steht folgende Theorie: Die Herzfasern besitzen das Vermögen, auf schädliche Reize mit Kontraktionen zu antworten; Zweck der Kontraktion ist die Entfernung der materia peccans oder die Überwindung eines Strömungswiderstandes.

Wepfers Doktorrede ist eine originelle Arbeit, die klinische Befunde mit Ergebnissen der medizinisch-biologischen Grundlagenforschung in fruchtbarer Weise verbindet. Eindrücklich betont Wepfer den Wert der empirischen Methode, der Beobachtung (autopsia) und der konkreten Erfahrung und experimentellen Verifikation (experientia) ${ }^{17}$. Die Arbeit bildet einen frühen Versuch, die Zirkulationslehre Harveys auf die Klinik und Pathologie der Herzleiden anzuwenden. Wepfers Schrift bedeutet einen Übergang von der traditionellen pneumato-humoralen Pathologie zur modernen Physiopathologie der Kreislauforgane.

\section{Anmerkungen}

${ }^{1}$ Wepfer, Johann Jakob, Disputatio medica inauguralis De palpitatione cordis, Basel 1647 (unpaginiert in XIII Thesen gegliedert). Zit. als De palpit. cord.

${ }^{2}$ Eichenberger, Pietro, Johann Jakob Wepfer (1620-1695) als klinischer Praktiker, Basel 1969 (Basler Veröffentl. z. Gesch. d. Med. u. d. Biol., Fasc. 26); idem: Autobiographisches von Johann Jakob Wepfer (1620-1695) in einem Briefwechsel mit Johann Conrad Brotbeck (1620-1677), in: Gesnerus 24 (1967) 1-23. 
${ }^{3}$ Eichenberger, Gesnerus 24 (1967), S.8f.: «Incidi tum in doctissimos huius sententiae [scil. circulationis sanguinis] propugnatores Clarum Dominum Veslingium, Johannem Leonicenum, Dominum Thomam Bartholinum ....

${ }^{4}$ Siehe Anm.3; Wepfer, J. J., Observationes anatomicae ex cadaveribus eorum, quos sustulit apoplexia, Schaffhausen 1658. In der Vorrede an den Leser geht Wepfer eingehend auf den vorbildlichen Einfluß Bartholins auf sein eigenes Schaffen ein.

${ }^{5}$ Galen, De tremore, palpitatione, convulsione et rigore liber, cap. 5 (Editio Kühn VII, 596).

${ }^{6}$ Sennert, Daniel, Institutiones medicinae, Paris 1632, S. 339 (Lib.2, pars III, sect. II, cap.2): «Cum alias videamus, quanto conatu et impetu cor moveri soleat, si rem aliquam molestam percipiat.» Siehe dazu Wepfer, De palpit. cord., Thesis III.

7 Temkin, Owsei, The classical roots of Glisson's doctrine of irritation, in: Bull. Hist. Med.38 (1964) 297-328.

8 Temkin (Anm. 7), S. 306-308.

${ }^{9}$ Wepfer, De palpit. cord., Thesis I; Thesis IV: «Palpitationem cordis a spiritu crasso, sive ventriculis, sive parenchymati incluso, non posse fieri, supra dictum est.»

${ }^{10}$ Wepfer, De palpit. cord., Thesis II: «Definiri potest palpitatio cordis, quod sit motus cordis pravus, vehemens, velox, creber, authore facultate eius irritata a re molesta, quam excutere conatur.»

11 Wepfer, De palpit. cord., Thesis II: «Praecipue hic constrictionis motus, quo cor in expellendo utitur, intelligitur, qui fit, rectis ventriculorum fibris apicem cordis basin versus, et transversis parietes eorum ad septum trahentibus, ut sinus intus angustiores evadant; quod Clarissimi Anatomici, rerum naturalium felices scrutatores, Columbus, Harveius et Celeberrimus Dn. Conringius de Gen. et Motu sanguin. cap. 36 observarunt: et quilibet eius rei veritatem sibi facile autopsia comparare potest, si vivens animal intueatur, parumque de cordis cono abscindat. Constrictionis actu conus etiam pectori appropinquare cernitur, qui illud procul dubio est, quod sub mamilla sinistra singulis ictibus emicat. Pulchre et manifeste aliquamdiu hoc in ranis animadvertere licet, si vivis corculum denudetur: in systole conus ad basin accedit, et pectori acutior appropinquat.»

12 Galen hatte schon die Herzexstirpation benutzt, um die Autonomie der Herzaktion zu demonstrieren; Galen, De placitis Hippocratis et Platonis, lib.6, cap. 3 (Editio Kühn V, 531); Galen, De anatomicis administrationibus, lib. 7, cap. 8 (Editio Kühn II, 614).

13 Wepfer, De palpit. cord., Thesis IV: «Cordi enim facultas pulsifica indita est, quae ab ipso ortum habet, non a cerebro communicata, nec in corde ebullientibus, vel in illud irruentibus humoribus tribuenda: quod non anxie conquisitis argumentis probari debet; sensus inspicientibus fidem faciet. Cor nempe corpore excisum, vacuum sanguine, per aliquod temporis spatium adhuc movetur ... et quilibet huius rei periculum facere potest, non solum piscium cordibus exemptis, quorum cordis motus per integrum diem perdurat, sed et quadrupedibus ... Peragit [scil. cor] autem motum insito calido, quo copioso perfunditur, et fibris ... quamdiu enim cor calet et fibrae continuantur, etiam corpore exemptum movetur; tenues vero in auras si calidum abiverit, et fibrae dissecentur, statim consueta agitatio cessat.»

14 Wepfer, De palpit. cord., Thesis IV: «Potentia praeterea instructum est cor, qua noxia et damnosa agnoscit, quae tactus est ... Utut sit, certe experientia cordi sensum inesse facile confirmabit, si cor nudum vel acu, vel alio instrumento pungatur; tum oculis usurpare licebit quos saltus facturum sit, quos conatus ad rem molestam repellendam adhibeat.» 
15 Thesis III, V, VI, VII. - Thesis III: «Causa proxima in singulis cordis partibus, et vicinis, subsistere potest, modo adverso stimulo ad cor pertingere valeat.» Thesis V: «Primum videlicet oportet, ut irritamenta assidue cor attingant.»

16 Thesis III, V, VI, VII.

17 Anm. 11, 13, 14.

\section{Summary}

Wepfer's inaugural disputation "On the Palpitation of the Heart" deals with a traditional topic with the new concepts and methods of seventeenth century experimental medicine. The treatise is based on the methods of anatomy, pathological anatomy and experimental physiology which are successfully related to clinical pathology. The palpitation of the heart is defined as a morbid, vigorous and frequent contraction of the cardiac fibers responding to irritation by adverse stimuli. Wepfer's doctoral disputation reflects the transition from traditional pneumatic and humoral pathology to the modern physiopathology of cardiovascular diseases.

Prof. Dr. med. Nikolaus Mani

Medizinhistorisches Institut

Sigmund-Freud-Straße 25

D-5300 Bonn 1 